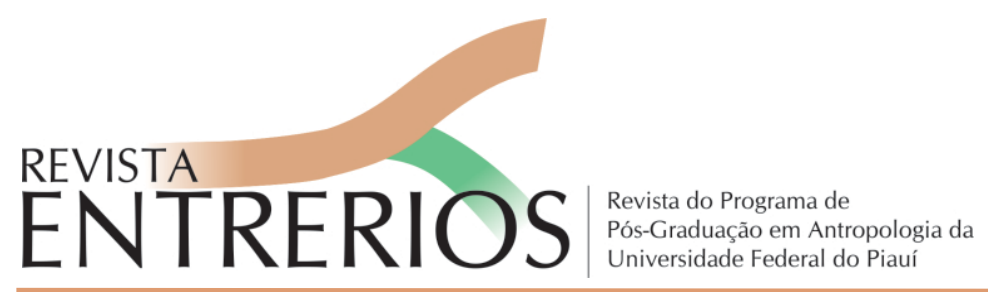

\title{
Los warao del Delta del Orinoco: aspectos de su cosmogonía, etnografía y situación actual
}

\section{Os Warao do Delta do Orinoco: aspectos de sua cosmogonia, etnografia e situação atual}

\author{
Omar Enrique González Ñáñez ${ }^{\dagger}$ \\ Douctor en Ciencias Sociales - Universidad Central de Venezuela (UCV) \\ Docente da Universidad de Los Andes, \\ Universidad Central de Venezuela y del Instituto Nacional \\ de Idiomas Indígenas-Ministerio del Poder Popular para la Educación \\ wamudana@gmail.com
}

\begin{abstract}
Resumen: El presente artículo ofrece una visión etnográfica del pueblo amerindio warao, una sociedad lingüisticamente independiente de aproximadamente 30.088 individuos (año 2011) que viven en el estuario del Delta del Orinoco, ubicado al noroeste de Venezuela, lugar donde dicho río desemboca en el Océano Atlántico, estando, además, muy próximo a la República de Trinidad y Tobago. En el texto se destaca aspectos mostrados por la etnografía tradicional respecto a la cultura warao, su importante cosmogonía, cosmología y algunos pasajes de su etnohistoria y mitología. Son mencionados también ciertos aspectos de salud, alimentación, economía y situaciones actuales sobre migración y éxodo de su hábitat no solo hacia el centro de Venezuela, sino a otros lugares como Guyana y Brasil.
\end{abstract}

Palabras clave: Sociedad hidráulica; cosmogonía; etnohistoria; chamanismo; salud indígena.

Resumo: Este artigo busca oferecer uma visão etnográfica do povo amerindio Warao, uma sociedade linguisticamente independente de aproximadamente 30.088 individuos (ano de 2011) que moram no Delta do Orinoco, no noroeste da Venezuela, onde esse rio deságua no Oceano Atlântico, lugar, aliás, muito próximo da República de Trinidad e Tobago. Destacam-se no texto do que na descrição etnográfica tradicional mostra da cultura Warao, sua importante cosmogonia, cosmologia e algumas passagens de sua etno-historia e mitologia. São mencionadas as questões de saúde, alimentação, economia e situações atuais sobre migração e êxodo de seu habitat, não apenas para o centro da Venezuela, mas também para a Guiana e o Brasil.

Palavras-chave: Sociedade hidráulica; cosmogonia; etno-historia; xamanismo e saúde indígena. 


\section{Pueblo warao}

Los warao constituyen una sociedad amerindia que podemos considerar una "civilización hidráulica" por su milenaria ocupación del estuario del Delta del Orinoco donde desarrollaron una sociedad hidráulica al estilo del antiguo Egipto o Mesopotamia (WITTFOGEL, 1953) en lo que hace a su ocupación, control y dominio de las aguas y antigua cultura (hace más de 20.000 años) asentados en algunos de los 36 caños del Bajo Delta del río Orinoco donde éste río desemboca en el Océano Atlántico. Nuestra cita de Wittfogel no tiene que ver con la construcción de obras hidráulicas en el Delta como en las civilizaciones orientales del Viejo Mundo ni busca aplicar el esquema evolucionista que usaron autores como el caso de Wittfogel, sino que se refiere al control de las aguas, no obstante, los warao aprendieron de sus vecinos lokono y chinos de Guyana, la construcción de "trinchas" y diques para el cultivo del arroz. Aunque estudios recientes demuestran que los arawaks (proto-lokono) habían construido en Guyana estructuras y canales de riego. Por ejemplo, en Berbice, Guyana, arqueólogos descubrieron una ciudad perdida de los proto-lokono (arawaks) con estructuras circulares y canales de regadío hechos de piedra.

Las investigaciones etnohistóricas y arqueológicas de Whitehead, Heckenberger y Simon en el área (2010) nos confirman este temprano desarrollo de una cultura hidráulica. Ellos señalan:

Future study to be initiated in 2011 builds on preliminary fieldwork conducted in the middle Berbice (NE Guyana) and the earlier work in the area by Whitehead and Simon (1991). In 2009, investigations were conducted at four occupation sites in a study area roughly $20 \times 10 \mathrm{~km}$ along the middle Berbice River. Three major episodes in the culture history of the region have been identified: 1) an early occupation of settled agriculturalists, dated to ca. $5000 \mathrm{BP}$, based on preliminary excavations at the Dubulay site; 2) a period of agricultural intensification, dated to ca. $1800 \mathrm{BP}$, associated with densely concentrated, small farming mounds; and 3) densely settled agricultural populations in early historic times (post-1540), associated with ancestors of the contemporary Lokono (Arawak) peoples, still present in the study area.

It has often been remarked of Guyanese colonial history that the Caribs were the natural occupiers of the middle and upper reaches of the Atlantic coastal rivers. However, we know now that they were driven there by expansive Arawakan colonization along the Atlantic coast from the Amazon to the south. Some of the later stages of this expansion, such as into the Pomeroon river, and the southern channel of the Orinoco River, was militarily assisted by the Spanish. Together they raided Carib settlements as part of their continuing alliance with the Lokono and formulated their regional policies with that alliance very much in mind (Ojer 1966, Whitehead 1988, 1990, 1994, 1995, 1997, 1998, 2003). Also related to this significant Arawakan presence is the existence, as in Brazil and Bolivia, of large scale earthworks and associated large scale settlement. The archaeology, history and ethnography thus, for once, appear to be in a perfect harmony over the persistent significance of an Arawakan macro-polity centered on the Berbice River in Guyana (WHITEHEAD, HECKENBERGER Y SIMON, 2010: 87-88) ${ }^{1}$

\footnotetext{
1 El estudio futuro que se iniciará en 2011 se basa en el trabajo de campo preliminar realizado en el centro de Berbice (NE Guyana) y el trabajo anterior en el área por Whitehead y Simon (1991). En 2009, se realizaron investigaciones en cuatro sitios de ocupación en un área de estudio de aproximadamente 20 x $10 \mathrm{~km}$ a lo largo del medio río Berbice. Se han identificado tres episodios importantes en la historia de la cultura de la región: 1) una ocupación temprana de agricultores asentados, que data de ca. 5000 BP, basado en excavaciones preliminares en el sitio de Dubulay; 2) un período de intensificación agrícola, fechado en ca. $1800 \mathrm{BP}$, asociado con pequeños montículos agrícolas densamente concentrados; y 3) poblaciones agrícolas densamente pobladas en los primeros tiempos históricos (posteriores a 1540), asociadas con antepasados de los pueblos contemporáneos de lokono (arawak), todavía presentes en el área de estudio.
}

EntreRios - Revista do PPGANT -UFPI -Teresina • Vol. 3, n. 2 (2020) 
En el territorio warao del Delta Amacuro, la única obra de ingeniería con el propósito de cerrar el caño Manamo a los fines de utilizar los terrenos de pasto para la cría de ganado (sobre todo en el vecino estado de Monagas), fue hecha por ingenieros criollos de la Corporación Venezolana de Guayana (CVG) a mediados de la década de los sesenta y fue una obra realizada sin consultar a sus habitantes, los warao.

\section{Ubicación etnográfica de los warao}

La sociedad warao ha sido objeto de investigaciones antropológicas muy sistemáticas, numerosas y densas, por ejemplo, los etnógrafos Werner Wilbert y Cecilia Ayala W. (2007) nos señalan que:

Los Warao fueron conocidos en la literatura histórica y antropológica de los siglos XVI-XX como Guaraúnos. Sin embargo, Lizarralde (1993: 124) recopila 54 denominaciones a manera de gentilicio, las cuales son el resultado de sinonimias (Guarao, Uarao, etc.), traducciones a otros idiomas (Warraw, Warrau, etc.), denominaciones de subgrupos (Winiquina, Mariusa, etc.) y calificativos foráneos (Faraute, Arote, etc.). No obstante, su autodenominación es la voz: warao. Su significado: "dueños de la canoa" (Wilbert J. en: Lizarralde 1993: 124), aunque Lavandero (1994: 18) sugiere "gente de playa" o "playeros". Su idioma se clasifica como independiente (Lizarralde 1993: 162), si bien se ha sugerido que podría estar relacionado con la familia lingüística Chibcha, pero esta filiación aún no ha sido aceptada como definitiva. Este pueblo habita principalmente en las islas y caños del delta del Orinoco en el Estado Delta Amacuro, Venezuela. Para el año 2011 -fecha del último Censo Indígena- su población se elevaba a 30.088 individuos, (subrayado nuestro) de los cuales el 88,61\% vive en el Estado Delta Amacuro (INE 2001). Sus principales actividades económicas incluyen la pesca y la cosecha de productos selváticos. Desde 1930 practican la horticultura y a mediados de la década de los cincuenta comenzaron a participar como mano de obra en las actividades productivas de las industrias agrícolas, pesqueras, madereras y las procesadoras de palmito, auspiciadas tanto por misioneros capuchinos como por entes particulares. Actualmente, la pesca comercial que se realiza en las lagunas y desembocaduras de los caños deltaicos está dirigida por el sector criollo desde Barrancas y Tucupita y sigue siendo, aunque itinerante, la ocupación principal del warao. La calidad de la data en todos los campos tradicionales de la antropología es excelente y muy calificada (Coppens, 1998: 925-929), pese a que la base literaria proviene mayormente del Municipio Antonio Díaz del Estado Delta Amacuro, por ser allí donde habita el 71,3\% de la población total warao" (WILBERT \& AYALA W., 2007:333-334)

Sobre la clasificación y etnonimia del pueblo warao, la etnóloga Jenny González Muñoz (2019) hablando de mitos fundantes en sociedades indígenas amerindias diversas señala que muchos de sus mitos

\footnotetext{
A menudo se ha señalado en la historia colonial de Guyana que los caribes fueron los ocupantes naturales de los tramos medio y alto de los ríos costeros del Atlántico. Sin embargo, ahora sabemos que fueron conducidos allí por la colonización de arawak a lo largo de la costa atlántica desde el Amazonas hacia el sur. Algunas de las etapas posteriores de esta expansión, como el río Pomeroon y el canal sur del río Orinoco, contaron con la ayuda militar de los españoles. Juntos asaltaron los asentamientos caribes como parte de su alianza continua con los lokono y formularon sus políticas regionales teniendo muy en cuenta esa alianza (OJER 1966, WHITEHEAD 1988, 1990, 1994, 1995, 1997, 1998, 2003). También se relaciona con esta importante presencia arawak la existencia, como en Brasil y Bolivia, de movimientos de tierra a gran escala y asentamientos asociados también a gran escala. La Arqueología, la Historia y la Etnografía, por lo tanto, parecen estar en perfecta armonía sobre el significado persistente de una macro-política arawak centrada en el río Berbice en Guyana.
} 
(...) Transcurre(n) fundamentalmente en relación con el medio acuático, tanto en lo que tiene que ver con la vivienda y la alimentación, como con la transportación (realizada en curiara, que es una embarcación estrecha fabricada con un solo tronco de árboles como el cachicamo o el carapo), causa por la cual se ha traducido a la palabra warao, etimológicamente hablando, como "gente de embarcación", "gente de curiara", o "gente de agua". Algunos, no obstante, aseguran que la palabra warao simplemente quiere decir "gente" (GONZÁLEZ MUÑOZ, 2014:3).

Citando al Padre (capuchino) Basilio Barral, ella escribe:

El verdadero nombre se lo han dado ellos mismos al definirse a sí propios guarao (warao) o guaraotuma (waraotuma) y también guarotu o guaraotu (warotu, waraotu), en contraposición de jotaro o jotarotu, con que se nombran a los no guaraos, criollos o indios. Ellos los no guaraos, son jotaraos (jota arao, gente de tierra). Ellos, los guaraúnos, no son jota arao (gentes de tierra), sino guarao (gua arao), gente de las embarcaciones, nautas de por vida (BARRAL, 1964 apoud GONZÁLEZ MUÑOZ, 2014:93).

Continúa la etnóloga González Muñoz (2014: 93):

Tal como lo devela la cita textual, la palabra warao o guarotu es utilizada por los miembros de dicha comunidad para distinguirse del resto de las personas que no son de su nación, indistintamente si son indígenas o no. En este sentido, el término warao no sólo debe significar "gente de embarcación", va más allá, hacia una aseveración que implica una vida total en franca conjunción con el agua del río, hasta el punto de distinguirse como excelentes profesionales en lo que a navegación se refiere, teniendo en cuenta que tal cercanía con el medio fluvial se circunscribe también a la fabricación de dichas embarcaciones como es el caso de la curiara".

Los asentamientos o aldeas warao se extienden al oeste de Tucupita, hacia los estados Monagas, Sucre y Bolívar donde también se les llama guaraúnos. En el río San Juan, estados Monagas-Sucre, existe una comunidad warao llamada Mosú (término criollo que resulta de la unión de Mo-Monagas y Su-Sucre).

Es necesario acotar que coincidimos con la posición de estudiosos contemporáneos de los warao como García Castro, Wilbert, Heinen y Gassón (2008) quienes consideran errado el término para referirse a esta sociedad como si fuese homogénea, porque más bien es una etnia con diferencias a nivel del Delta, (warao de tierras altas y warao de tierras ribereñas bajas o "morichaleros") (GARCÍA CASTRO \& HEINEN, 2013) así como con influencias de pueblos indígenas vecinos de habla y cultura tanto caribe (ye'kuana, makuxi) como arawak (lokono y mapidian de Guyana); podría hablarse de un pueblo multiétnico. También encontramos comunidades warao mezcladas con pueblos criollos (jotarao en idioma warao) como los que habitan en Guayos o en Tucupita, Barrancas o San Félix. Los análisis sobre el tipo de sus viviendas y de sus alimentos es un buen indicador de esta mezcla.

Los warao están culturalmente identificados por la recolección de la palma de moriche (mauritia flexuosa) a la cual consideran "el árbol de la vida" pues el moriche (ojidu aroku) tiene usos múltiples en esta sociedad, no solo para la construcción de sus viviendas (el techo y paredes) en la región suroriental del Bajo Delta pues en la costa noreste del Delta lo que se consigue es el temiche (yawiji aroko) que es la palma que emplean en sus casas. El moriche les provee además de un almidón con el cual hacen tortas de almidón; asimismo en el moriche se cría un gusano que los warao comen, también el moriche lo utilizan para el tejido de cestas. 
En este trabajo solo mencionaremos tangencial o periféricamente los problemas de salud y migración que han dado a conocer más a este pueblo que los aspectos de su perfil etnográfico; especialmente durante los últimos tres años que se ha producido el desalojo y desplazamiento de sus caños ocasionado tanto por la invasión criolla como por la desatención y falta de políticas públicas hacia ellos y, por las últimas epidemias y enfermedades que los han diezmado como los casos de las más críticas como el cólera, el SIDA, tuberculosis, diarreas, malaria e incluso desnutrición, lo que ha desencadenado una migración de carácter internacional especialmente hacia la vecina República Federativa del Brasil, tema que será abordado exhaustivamente por otros colegas en este número especial de la revista "Entreríos".

\section{La Salud entre los warao: la visión occidental y la perspectiva warao}

Para este aspecto consideramos como lo más pertinente retomar las investigaciones de los expertos en etnomedicina. Por ejemplo, Werner Wilbert y C. Ayala Lafée (2007:333):

\section{"Salud"}

Aparte de las enfermedades epidémicas que azotan la región (sarampión, cólera y tuberculosis) la salud del pueblo warao varía de acuerdo al estilo de vida, que aquí categorizamos como perteneciente a tres formas de vida:

1) Aquella en la que su alimentación se basa en los derivados proteínicos del pescado y en el ocumo chino como fuente de carbohidratos. Generalmente, los Warao que se alimentan de esta manera son aquellos que habitan en las comunidades más alejadas y mantienen su entorno cultural tradicional. No tienen acceso frecuente a los productos occidentales. Su salud podría compararse con los niveles socioepidemiológicos de hace unos ochenta años. 2) Aquellos otros que se empeñan en comprar sus alimentos "en la bodega" para dejar de ser "indio" o persona "de segunda clase". Sin embargo, éstos no cuentan con los recursos económicos necesarios para comprar lo que requiere una dieta balanceada. Los productos que ofrecen las bodegas se limitan a harinas, espaguetis, refrescos y unos pocos enlatados. Entre éstos, se observa un marcado decaimiento en los niños, una relativamente alta incidencia de enfermedades infecciosas y una depresión psicológica causada por una frustración sostenida en el tiempo, que se traduce en un incremento de riñas entre familiares. El resultado más importante de esta situación es el deterioro de la salud, especialmente en el sector no productivo de la población (niños, ancianos y enfermos). Las comunidades en que habitan están sufriendo una ruptura sociocultural. En éstas se observa a los adultos mayores y ancianos luchando para mantener la cohesión tradicional del grupo contra la resistencia de los adolescentes y adultos jóvenes quienes, en su afán de emular la vida criolla, han reorientado sus deberes para con la familia extendida sólo hacia sus familias nucleares. 3) La de aquellos otros que habitan en comunidades desarrolladas por organizaciones gubernamentales o religiosas. Estas comunidades cuentan con luz eléctrica, escuelas, comedores, bodegas, medicatura y capilla. Por asociación, allí se encuentra la tasa más alta de empleos fijos (jardineros, planteros, concejales, transportistas, mecánicos, carpinteros, cocineros, policías, comisarios, enfermeras y enfermeros, etc.) y, por tanto, una mayor independencia económica. La gran mayoría de los adultos jóvenes han dejado las actividades de la pesca y el cultivo para subsistir exclusivamente de sus salarios. Sin embargo, a pesar de que en la propia localidad se ubica un Centro de Atención Integral 2 2, en el cual los médicos pueden controlar los trastornos cotidianos, los ingresos de este grupo tampoco alcanzan para alimentarse balanceadamente, observándose una alta tasa de obesidad, una gran acumulación de deshechos sólidos con la consiguiente presencia descomunal de ratas, y un elevado consumo de alcohol entre sus po- 
-bladores. El número de casos atendidos por el CAI 2, a causa de situaciones motivadas por accidentes y riñas provocadas por el alcohol, son numerosos".

Wilbert y Ayala Lafée (2007:363) añaden:

\section{Concepción de la salud}

El concepto de salud (bajuka) en la etnomedicina warao es la condición fisiológica "sin olor" del individuo, la cual es causada y mantenida por la disposición de su sangre. Así, el estado de la sangre influye directamente sobre la calidad de la salud del individuo. En el transcurso de la vida la salud se ve amenazada por patógenos (olores fétidos) que invaden el cuerpo cuando la sangre se encuentra en un estado "débil" (Wilbert, W. 1996: 202). Para el warao, el cuerpo humano está lleno de sangre, del mismo modo que un árbol lo está de savia. Tanto los hombres como las mujeres portan una cantidad finita de sangre, aun cuando consideran que la de los varones es más fuerte que la de las hembras, debido a los diferentes grados de resistencia que muestran los dos sexos frente a tareas extenuantes. Sin embargo, es la fuerza relativa de la sangre de cada individuo lo que determina la susceptibilidad a la enfermedad, ya que ésta puede fluctuar considerablemente y funcionar "muy por debajo" de su capacidad potencial. Los Warao creen que para mantener la sangre fuerte el individuo debe alimentarse apropiadamente, pues el alimento al filtrarse en el cuerpo se ablanda y vitaliza la sangre antes de la excreción. La sangre fuerte es roja y "acuosa" (joto taera) y la asocian a un buen estado de salud (Wilbert, W. 1996: 204). La sangre negra (joto ana), tal como la observan en las excretas de algunos enfermos, se considera débil y la relacionan a la enfermedad. La sangre coagulada (joto akoba) es considerada como sinónimo de muerte (Ayala y Wilbert 2001: 220). Por medio de la relación sexual la sangre del varón y de la hembra se unen para formar el feto. La concepción, sin embargo, sólo es posible si la sangre de la pareja es compatible, es decir, si la calidad de ambas está a su máxima potencia. Sin embargo, la calidad relativa de la sangre de la hembra no debe sobrepasar a la del varón. Para un warao, la pérdida de sangre es un acontecimiento altamente riesgoso, puesto que disminuye la vitalidad y la capacidad reproductiva de la víctima, exponiéndola a la enfermedad, es decir, a la posibilidad de que el estado fisiológico "sin olor", sea invadido por un olor fétido.

\section{Epidemias}

En términos de epidemias relativamente recientes entre los warao, quisiéramos mencionar la del cólera, flagelo que azotó al pueblo warao asentado en el Delta central y el Bajo Delta en el 2007. Afortunadamente para ese tiempo estaban en la zona una pareja del área de salud indígena integrada por los esposos Charles Briggs (antropólogo) y la médica Clara Mantini de Briggs (2015) quienes, aparte de su investigación-acción terapéutica han publicado dos libros con importantes testimonios sobre la epidemia, aparte de contar en su misión con apoyo directo del ministro de Salud para la época (Dr. Francisco Armada) y un excelente equipo de enfermeros warao y de expertos asesores en el área de salud indígena. Los textos son: Las historias en los tiempos del cólera (2004) y Una enfermedad monstruo. Indígenas derribando el cerco de la salud (2015), escrito por Charles Briggs, Clara Martini-Briggs, Norbelys Gómez, Tirso Gómez, Conrado Moraleda Izco, Enrique Moraleda Izco.

Del segundo texto (2015) que se basa también en testimonios de los warao afectados, investigadores de la enfermedad y de etnomédicos y enfermeros warao, recogemos algunos párrafos de su "Informe presentado en Caracas" (apéndice I del libro): 
A través de una investigación realizada por un equipo interdisciplinario conformado a petición expresa de líderes warao se contactó con testimonio de miembros de comunidades, familiares de los fallecidos, personal médico, de enfermería y misioneros quienes atestiguaron su conocimiento de las defunciones en la población warao desde el año 2007.

La investigación arrojó un total de treinta y ocho (38) defunciones de venezolanos, miembros de la etnia warao. Esta investigación se conformó con carácter de urgencia para recopilar información acerca de las muertes que mantienen a los vwnwzolanos miembros de la etnia warao en un estado de angustia y miedo por sus vidas y la de los suyos. La misma fue realizada en treinta (30) comunidades del Bajo Delta, parroquias Manuel Renaud y Padre Barral, en un tiempo de cinco días.

Esta enfermedad aparentemente de diagnóstico clínico según diagnóstico de las autoridades regionales de medicina, autoridades practicantes de la medicina warao y la propia comunidad. Tampoco se conoce diagnóstico de los resultados de los exámenes realizados a los fallecidos como por ejemplo muestras de sangre y autopsia.

Los síntomas de este cuadro clínico reportados: dolor de cabeza, fiebre, malestar general, dolor de estómago y dolor torácico, sensaciones sensoriales alteradas como hipersensibilidad al tacto de cualquier parte del cuerpo, picazones en los pies, parálisis de miembros inferiores de forma ascendente hasta miembros superiores, dificultad para tragar sólidos y líquidos, hidrofobia (miedo al agua), salivación espesa (a veces acompañada con vómitos) y termina con convulsiones y rigidez espástica, al momento de la muerte. La mayoría de los pacientes mueren en estado consciente hablando o solo llorando en caso de pacientes del primer año de edad...

Este cuadro clínico corresponde estrechamente a la enfermedad infectocontagiosa denominada de rabia que por el antecedente de mordidas de murciélagos nos hace presumir el diagnóstico de rabia selvática (subrayado nuestro) (BRIGGS et al. 2015:181-187).

Antes de referirnos al tema de la Cosmogonía y Cosmovisión warao, quisiéramos comentar brevemente la situación actual de la Educación Propia Indígena Warao (EPI-W) y la Educación Intercultural Bilingüe (EIB), la cual se implantó en Venezuela el 20 de septiembre de 1979, mediante Decreto Presidencial Nº 283 de 1979. Es decir, es una legislación que ya cumplió casi medio siglo y aunque pretendía potenciar y recuperar la educación originaria indígena lo que hizo fue sustituirla por la educación criolla; algo parecido a la educación que habían impartido las misiones religiosas, pero con mayor control del Ministerio de Educación. Frente a esa política, desde el mismo Ministerio se implementaron en el 2014, los “Proyectos Educativos por Pueblos Indígena" (PEPI) tratando de devolver a estos pueblos el manejo y construcción de su educación como siempre había sido, solo que con apoyo financiero y reconocimiento institucional del Ministerio del Poder Popular para la Educación. A pesar de la supuesta autonomía basada en modelos autóctonos o propios, el Estado ha insistido en manipular ese proceso. En el caso warao, a través de la Dirección de Educación Intercultural del MPPE y la Zona Educativa de Tucupita, se está concluyendo el PEPI Warao (coordinado por el Sociólogo José Farías y el profesor warao Eufrasio Jiménez). Está por verse cómo se implementará el Proyecto y qué papel jugarán los líderes y el movimiento organizado warao en su aplicación y cuál será su nivel de autonomía. 


\section{Cosmogonía: El Universo mítico y religioso del pueblo warao}

Aun cuando algunos criollos respetan todavía sus creencias y prácticas religiosas, la mayoría de la población no indígena las desconoce y a veces la discriminan y atacan (GARCIACASTRO \& HEINEN, 2000), a pesar de lo temprano que comenzó la evangelización cristiana entre ellos con su consiguiente etnocidio. Sobre la evangelización foránea tomamos una cita de García Castro \& Heinen (2013:18):

Hay que apuntar que los misioneros Capuchinos llegaron en 1919 a Tucupita después de haber estado en Venezuela desde 1891 (Martín, Elías, 1977: 8, 13). Y aunque según Margolies llegaron de España en 1922 (2006:155), luego reconoce que con la Ley de Misiones de 1915 la Orden Capuchina tenía jurisdicción política, económica y civil sobre los warao de los territorios federales especificados en su contrato (Margolies, 2006:157). La razón que para ello tuvo el Dictador de entonces, Juan Vicente Gómez, era obviamente la de frenar la usurpación de territorio por parte de Ingleses y Brasileños en la Gran Sabana al sureste del país. En 1924 se efectuó "la restauración de las Misiones", con la fundación de la Misión de Araguaimujo (Arawaimuju) en la frontera entre las plantaciones criollas con la tierra indígena Warao.

El Padre E. Martin en 1977 nos habla de la presencia de más de 50 años de los misioneros capuchinos entre ellos. Algunos misioneros han hecho importantes investigaciones sobre sus creencias y cultura e incluso han sido coautores conjuntamente con antropólogos, de algunos textos etnográficos indispensables sobre los warao (LAVANDERO \& HEINEN, 1986).

Hablando de la salud warao en términos de su cultura y su percepción, Wilbert y Lafée en la obra citada, nos precisan lo que ellos llaman "La salud en su cosmovisión":

La cosmovisión warao asume que la salud es el estado normal del individuo. Sin embargo, enfatiza que la fuerza física, resistencia y capacidad reproductiva dependen de la calidad de la sangre del individuo. Especifica que la dieta adecuada se basa en el consumo del pez osibu (morocoto), del aru (yuruma), del oji (fruta de la palma moriche) y del mo (larva del escarabajo) alimentos asociados con esta palma. Asimismo, indica que sólo los niños sufren de enfermedades "naturales", pues la salud de los adultos se debilita exclusivamente por reprobaciones o maldiciones místicas que producen síntomas parecidos a los que causan las enfermedades naturales. De hecho, también revela que ningún adulto muere por causas naturales sino por el ataque de un jebu como resultado de infracciones cometidas por la víctima contra las normas cosmológicas, detalladas anteriormente. Por consiguiente, la salud del individuo depende directamente de su propio comportamiento en el entorno socioambiental (WILBERT Y LAFÉE, 2007:364-370).

Respecto al chamanismo, afirma que

El chamanismo entre los Warao es una manifestación mágico religiosa plasmada en una fisiología natural, reafirmada por ceremonias y parafernalia adaptada al medio ambiente natural y transformación cultural del delta del Orinoco, pese a que en su contexto universal las técnicas sagradas y dialécticas convergen hacia un chamanismo puro que difiere poco del que se practicó antiguamente en el Asia, desde donde procedió. En la práctica del chamanismo warao existen rasgos generales comunes al Viejo y Nuevo Mundo: tendencia espiritual, incorporación de entes sobrenaturales a manera de asistentes, rocas que simbolizan el alma de espíritus familiares, enfermedades enviadas a través de saetas mágicas. 
Igualmente, hallamos paralelismos cósmicos en los cuales el chamán transita en viajes etéreos por los diferentes mundos habitados por entes o deidades cardinales; la convicción en la existencia del cosmos de arriba, del inframundo, del mundo del oeste y del este; igualmente, el poder sobre la vida y la muerte y el mecanismo de la curación o recuperación de la salud. El humo del tabaco es el vehículo que conecta al chamán con el mundo cosmológico; es una especie de cordón metafísico que se tiende entre él, los espíritus y los dioses ultraterrenos. Entre los dominios sobrenaturales existe un orden jerárquico que guarda relación con la virulencia de la enfermedad que cada uno de los dioses domina. En este sentido, el dios del norte, Warawaro (la mariposa), envía ventoleras heladas enfermando a los Warao con dolencias respiratorias como la "tos del mono aullador", tos ferina (Jebu waiobo), neumonía (Jebu obo sabana), etc. El dios del sur produce las enfermedades febriles como el sarampión (Jebu bororo) y el paludismo (Jebu tororo). El dios cardinal del este controla las enfermedades gastrointestinales como el cólera (Jebu obonona asida) y la disentería sanguinolenta (Jebu sojoto). Por último, el dios del oeste envía la muerte a través de enfermedades hemorrágicas como la tuberculosis pulmonar (Jebu obo monida) (Wilbert, W. 1996: 19). Las enfermedades son tratadas por tres clases de chamanes (bajanarotu, joarotu y wisiratu), quienes actúan como intermediarios entre los seres místicos y los seres humanos. El bajanarotu obtiene su poder del "Espíritu del Tabaco" y de Mawari, deidad representada por un ave mitad gavilán y mitad tijereta, relacionada con agudas crisis de salud y muertes repentinas. Lleva en su pecho a dos de los "hijos" (jebu) de este espíritu que actúan como entes tutelares, los cuales pueden salir por sus brazos como dardos y causar enfermedades o la muerte. Este chamán aplaca a los seres místicos con humo de tabaco. Entre los bajanarotu, el de mayor rango es el Daunonarima, el Guardián del Maniquí de Madera - Daunona, manifestación del Espíritu del Tabaco, objeto sagrado que el chamán puede enviar para producir brotes epidémicos de gastroenteritis. Las prácticas del bajanarotu, como intermediario y curandero, se relacionan con enfermedades gastrointestinales, las cuales puede prevenir, causar o curar (Wilbert, W. 1996: 20). El joarotu, o "dueño de la joa", se alinea con Joebo el dios cardinal caníbal del inframundo en el oeste. Lleva en su pecho a dos de los hijos de esta deidad, quienes a su vez, se encargan de hacerle cumplir sus promesas iniciáticas de que por medio de la hechicería deberá proveerlo a él y a su corte de sangre y carne humana. Si el chamán niega su obligación, los seres místicos del poniente destruirían a su gente y ellos mismos llegarían a su fin. El "sacrificio humano" por medio de la hechicería es indispensable para la permanencia de su gente, y periódicamente el joarotu está obligado a usar su honda mágica y sus dardos de humo de tabaco para matar por este medio a alguna persona de una comunidad vecina. La deidad protectora del joarotu expresa su agradecimiento dotando a su servidor con el poder de curar las enfermedades que van acompañadas de pérdida de sangre, incluyendo la disentería sanguinolenta que representa el mayor flagelo para la vida humana en el delta del Orinoco (Wilbert, W. 1996: 20). El wisiratu, o dueño del dolor, es el chamán o sacerdote étnico que sirve de mediador entre su pueblo y el Jebu. Detenta el poder de los dioses del norte, del este y del sur. Desde el momento de su iniciación, lleva en su cuerpo seis hijos de estas deidades cardinales que le sirven de entes tutelares. Durante toda su vida le recuerdan los votos que hizo en su encuentro iniciático de proveer periódicamente a los dioses y a su corte de humo de tabaco y de sagú de moriche. A cambio, los dioses disminuyen la muerte de niños y dotan al wisiratu con el poder de curar enfermedades. Aparte de sus funciones como curandero, el wisiratu también proporciona orientación psicológica y refuerza las normas morales dentro del grupo. El wisiratu de mayor rango es el Guardián de la piedra Sagrada, manifestación de la deidad tutelar para sus seguidores y, a la vez, su 
principal fuente de protección (ibid.: 21) Entre este grupo debe de incluirse a la fitoterapeuta, pese a que sus poderes son más bien seculares. La práctica de la fitoterapeuta (yarokotarotu) en la comunidad warao no es ritual, aunque se realice dentro del marco religioso. Pero el hecho de que definamos esta práctica como "no ritual" no significa que sea una práctica secular, ya que la fitoterapeuta reconoce que los agentes de las enfermedades endémicas son de naturaleza metafísica y que su praxis y sus medicamentos forman parte del sistema religioso warao. Sin embargo, la fitoterapeuta no está sometida a una iniciación de tipo chamánico antes de llegar a ser una experta; como tampoco están sujetas a ningún ritual las actividades de recolección, preparación y administración de las medicinas botánicas (idem). La fitoterapeuta trata tres clases de enfermedades no místicas. Éstas incluyen: 1) enfermedades gastrointestinales (diarreas, vómitos, dolores de estómago, etc.); enfermedades respiratorias (gripes, tos); enfermedades febriles y cutáneas que se creen son causadas por la intrusión de un olor fétido en el cuerpo humano; 2) enfermedades debidas a "sangre débil", trastornos del sistema reproductor de la mujer y pérdida de energía por esfuerzos excesivos y la prolongada exposición a los elementos naturales (lluvia, viento, frío, calor, etc.); y 3) enfermedades causadas por parásitos intestinales, picaduras de escorpiones, arañas, culebras, etc. y trastornos odontológicos (WILBERT Y LAFÉE, 2007: 2122).

En un artículo reciente que hicimos a Noreye Guanire, Anairamiz Aranguren e Gonzales Nañez (2008), explicamos la práctica del Wisidatu o chamán warao.

El Warao utiliza diferentes técnicas curativas como por ejemplo: la quema de restos de materiales y animales, estos produce olores fuertes ahuyentando a los malos espíritus que provocan las enfermedades. Esta es una de las diferentes técnicas de medicina originaria utilizada por el Warao: tradicionalmente cuando enferma un Warao se busca el Wisidatu, yerbero, maraquero "Marimataro", en la actualidad se lleva a la medicatura para que le suministre tratamiento "yarokota" mientras que otro sector indígena Warao prefiere la practica visionaria del Wisidatu, la "Chamanica". El Joarotu: El poderoso, el más grande en el mundo del mal y el bien (Joá): El amo de la brujería. Entre la etnomedicina, cosmovisión y práctica curativa los primeros tratamientos que recibe generalmente el enfermo es el reposo en su chinchorro que lo une a su ombligo como un hilo delgado y fino en su existencia entre la tierra y el cielo. Este, después de un baño frío le pasa la fiebre. Se le prohíben algunos alimentos en particular la carne y el pescado, el enfermo debe tomar brebajes preparado con hojas y tallos tiernos de clavellinas, también se le puede frotar todo el cuerpo con esta hoja e invocar a los espíritus sanadores con lo que el enfermo experimenta una sensación de frescor. Es importante discernir en el contexto de la practica simbólica estética, ritualista en donde el Wisidatu se conduce a un sistema de curación como la de succionar para eliminar el mal que aqueja el cuerpo - mente - espíritu a través de una realidad tanto simbólica como alternativa, viaje visionaria de los espíritus, alternativa para rescatar un alma. La interpretación de la enfermedad y el valor de las técnicas de la medicina moderna. Pero, en la totalidad de los casos de curación del Wisidatu y el Joarotu, a nivel psíquico o espiritual se considera conectada a la medicina occidental, como una opción al hombre con una carga valorativa como los sentimientos que son compartidos por las creencias, lo mitológico, lo simbólico (GUANIRE, ANAIRAMIZ ARANGUREN E GONZALES ÑAÑEZ , 2008: 3). 


\section{Cosmogonía}

Los otros aspectos que consideramos fundamentales y clásicos de los saberes warao refieren a su cosmogonía y los mitos y narrativas vinculados con este episteme, los cuales fueron analizados pioneramente por Johannes Wilbert (1979). Allí inserta dos referencias a lo que él llama "Mythical Geography" o geografía mítica, donde cita el concepto cosmológico de los warao del área central, y concretamente los sub-tribus de Mariusa, Winikina y Arawabisi. Esa área vinculada con el llamado territorio Hoanarao (gente de las aguas negras) quienes le expresaron que ellos "estaban en el centro" del espacio cósmico, practican el culto a la piedra sagrada. Esta expresión se debe, según Wilbert, a que el entorno geográfico de esa área donde viven los warao, no posee montañas y por tanto, el panorama está delimitado muy hacia el sur por la cadena montañosa de la Sierra de Imataca y hacia el norte por un promontorio ubicado en la isla de Trinidad, en el lugar llamado Naparima. Por tanto, para los warao "la tierra tiene forma de disco, siendo su suelo muy delgado y el cual flota sobre el océano". La causa de que la tierra flote se debe, según esta concepción cosmológica warao, a que la sostiene "firmemente en su centro, un monstruo serpentino terrestre, cuyas cuatro cabezas apuntan en dirección a los cuatro puntos cardinales", afirma Wilbert.

Para finalizar acotamos lo planteado por los investigadores Dieter Heinen y Rafael Gassón (2006), del Instituto Venezolano de Investigaciones Científicas (IVIC), quienes recogen y retoman el importante trabajo de Wilbert en su artículo "El verdadero Delta Indígena: Ecología etnohistórica del Delta del Orinoco".

\section{Consideraciones finales: Los cuatro cuadrantes del Universo Warao}

Johannes Wilbert (1979) publicó un artículo fundamental con el título Geography and Telluric Lore of the Orinoco Delta, en el que presentó el Universo Warao dividido en cuatro secciones, cada una con poblaciones diferentes. Vale la pena mencionar que Heinen (1988) trabajó datos etno-económicos del Delta que en puntos interesantes coincidieron. En esto Heinen y Rafael Gassón (1966: 61-66) dicen que

Los Warao ya no viven en los cuadrantes originales de tiempos prehispánicos ya que en los tiempos agitados de la colonia temprana y hasta el siglo XVIII muchos se refugiaron en el área de los Waharaowitu y sus morichales. Hoy en día, la situación es un mosaico de subgrupos cada uno con sus particularidades en rituales (naja namu, nowara), fiestas (mare mare) e instrumentos musicales (seke seke). La diferencia más grande es entre los morichaleros (daunarao, ojidunarao), básicamente sin curiaras, y los moyomo (tales como los Siawani), carpinteros de ribera.

Entre estos últimos el Universo Warao se extiende más allá del cerro Karosimo apuntado por Wilbert hasta la Altoplanicie de Nuria en la Serranía de Imataca donde localizan Wajakarima, el "Guardián de Tierra Firme" contrapuesto con Anabarima, "Guardián de las Olas", ambos en 61o27' longitud Oeste. En la tradición mitológica Warao cada una de los cuadrantes está asociado con diferentes grupos humanos con diferentes modos de subsistencia: cazadores de váquiros en el sureste del Delta y en el área del Amacuro, mezclados con Lokono de habla arawaca; pescadores en el noroeste que conocían la explotación del sagú y la fabricación de canoas, habilidades que transmitieron a los recolectores Waharaowitu en el noreste; y finalmente conuqueros Warao mezclados con indígenas de habla caribe en el suroeste. Tal como apunta Wilbert, "el mito expli- 
-ca los recursos naturales y la forma correspondiente de explotación" (1993[1979]:16). El mito Warao del héroe tribal Jaburi nos demuestra esta tradición (Roth 1908-9: 120, 122-5). Jaburi, el portador del arte de hacer curiaras nació en el cuadrante sureste, de donde era su padre Mayara-koto, el asador (de váquiros), a quien mató un espíritu "tigre" (Roth 1908-9: 122-3, Wilbert 1964: 28). Las madres (danituma) de Jaburi fueron dos hermanas de la parcialidad de los Siawani, quienes sabían como procesar aru, la harina de moriche (ojidu aru) (Wilbert 1964: 29). En la versión del mito relatado por Barral (1960:41-2) Mayakoto es un pescador. Lo mató una serpiente mitológica, Jajuba. Sin embargo, en esta versión hay dos Jaburi, un hijo de cada una de las dos mujeres. La versión de Barral también habla de yuca y de una casa cerrada por todas partes donde los horcones y los tabiques no hablan Warao. Sin embargo, el lorito se lo cuenta en Warao a la rana Wauta. En varias versiones del mito, la gente nutria o perros de agua, joetobu, son los tíos (hermanos de la madre) de Jaburi. Ellos son los Siawani o Chaguanes que los Waraowitu consideraron como caníbales, pero también como portadores de habilidades técnicas tales como el procesamiento de sagú y la fabricación de canoas (WILBERT, 1964:141-3).

(...) Wilbert ha trazado el camino tomado por Jaburi desde el cuadrante del cazador, a través de la tierra de Wauta y, después de inventar la canoa con sus canaletes, a lo largo del caño Macareo hasta Nabarima en Trinidad (1993: 14-15). Se puede especular que cada una de las poblaciones de los cuadrantes tiene un animal totémico, en el sentido amplio del término. Ya hemos visto que los Waraowitu se identifican con wajaromu, el ave playera Tiwi-tiwe. Lavandero piensa que el baile del Pájaro Guarandol que se encuentra en todo el Noreste de Venezuela y en la isla de Margarita, corresponde a wajaromu (Lavandero, comunicación personal). El cuadrante en el Noroeste del Delta corresponde a los "perros de agua", los Siawani, mientras que los Kari'ña son conocidos como "tigre" o jaguar. Los Lokono de habla Arawaca tienen clanes con sus respectivos tótem, pero no se conoce ninguno que abarque el total de dicha etnia. Tal como ya hemos apuntado arriba, los Guayanos, vecinos de los Waraotu del estado Sucre puede que sean la gente iguana. Ellos, seguramente de habla caribe, eran idénticos a los Pariagoto de la península de Paria donde se encuentra hoy el poblado de Guayana, (ver Carrocera 1979:XIV-XV) de donde proceden. Puede que Wayana sea la iguana (en Warao yowana) ya que Robert Harcourt menciona como su nombre "Guayna" o "Guyana" (Harcourt [1613] 1967: 151). Sin embargo, esto hasta la fecha solo es una conjetura (HEINE \& GASSÓN, 1966: 6166).

Como plantean los autores sobre la importancia y vigencia de la investigación de Johannes Wilbert (1979), la cosmogonía y cosmovisión del universo para los warao y su plano se mantienen en su imaginario a pesar de la penetración y aculturación ejercida sobre ellos por los jotarao u occidentales, incluidos los misioneros religiosos.

\section{Referencias bibliográficas}

BARRAL, BASILIO DE. Guarao guarata: Lo que cuentan los indios Guaraos. Caracas: Escuelas Gráficas Salesianas. 1960.

BRIGGS, L. Charles, MARTINI-BRIGGS, Clara. Las historias en los tiempos del cólera. Caracas: Nueva Sociedad, 2004. 
.; MARTINI-BRIGGS, Clara; GÓMEZ, Norbelys; GÓMEZ, Tirso; MORALEDA IZCO, Conrado; MORALEDA IZCO, Enrique. Una enfermedad monstruo. Indígenas derribando el cerco de la discriminación en salud. Buenos Aires: Lugar editorial. Edil, 2015.

GARCÍA-CASTRO, Álvaro; HEINEN, Dieter. Las Cuatro Culturas Warao. In: Tierra Firme. Revista arbitrada de Historia y Ciencias Sociales, n. 71, julio-septiembre, Caracas, 2000.

.; HEINEN, Dieter. Arquitectura Indígena Venezolana y Heterogeneidad Warao. Una Aclaración Necesaria In: Boletín Antropológico, año 31, n. 85, enero-Junio, 2013.

GONZÁlEZ MUÑOZ, Jenny. Mitos fundantes en la fuerza espiritual de los warao de Venezuela. In: Património e Memória, São Paulo, UNESP, v. 10, n. 2, p. 91-106, julho-dezembro, 2014. Disponible en: http:// pem.assis.unesp.br/index.php/pem/article/view/409

Etnia indígena warao: visibilidad de los prejuicios occidentales contemporáneos hacia la ancestralidad. In: Revista Serviço Social e Saúde. UNICAMP, vol. 18, 2019. Disponible en: https:/ / periodicos.sbu.unicamp.br/ojs/index.php/sss/article/view / 8656931/22177 DOI:https:/ / doi.org/10.20396/sss.v18i0.8656931

GUANIRE, Noreye; ARANGUREN, Anairamiz; GONZÁLEZ ÑÁÑEZ, Omar. Wisidatu: Mágico espiritual de los indígenas warao de Tucupita y de la Isla Araguabisi en el Estado Delta Amacuro. In: Boletín Antropológico, vol. 26, núm. 73, mayo-agosto, Mérida: Universidad de los Andes, p. 149-172, 2008. Disponible en: http:/ / www.saber.ula.ve/handle/123456789/30450.

HARCOURT, ROBERT. A Relation of a Voyage to Guiana. Appendix I: Collection of the passages containing fresh matter in the Second Edition of Harcout's Relation. Edited with Introduction E Notes by Sir Alexander Harris. Nendeln, Liechtenstein: Kraus Reprint Limited, [1613]1967.

HEINEN, Dieter. "Los Warao", en Los aborígenes de Venezuela, editado por Walter Coppens. Caracas: Fundación La Salle. 1988.

.; GASSÓN, Rafael El verdadero Delta Indígena: Ecología etnohistórica del Delta del Orinoco. In: Revista Copérnico. Año III, n. 5, julio - diciembre, p. 61-66, 2006. Disponible en: http:/ / www.revencyt.ula.ve/storage/repo/ArchivoDocumento/copernico/v3n5/art6.pdf

.; GASSÓN, Rafael (Edit.). Forasteros en su propia tierra. Testimonios de los Amerindios Warao. Caracas: Ediciones IVIC, 2008.

LAVANDERO, Julio; HEINEN, Dieter. Canciones y bailes del ritual de la Nouara. In: Montalbán, n. 17, p. 199-243, 1986.

MARTÍN, Elías. En las Bocas del Orinoco: 50 años de los misioneros capuchinos en el Delta Amacuro 1924-1974. Caracas: Ediciones Paulinas, 1977.

ROTH, WALTER E. An inquiry into the animism and folklore of the Guiana Indians. 30th Annual Report. Washington D.C.: Smithsonian Institution,1908/1909.

WHITEHEAD, Neil L.; HECKENBERGER, Michael J.; SIMON, George. Materializing the Past among the Lokono (Arawak) of the Berbice River, Guyana. In: Antropológica, Tomo LIV, n. 114, p. $87-127,2010$. 
WILBERT, Johannes. Warao oral literature. Caracas: Fundación La Salle (ICAS), 1964.

5(1):129-150.1979.

"Geography and Telluric Lore of the Orinoco Delta", en Journal of Latin American Lore Mystic endowment: religious ethnography of the Warao Indians. Cambridge, Mass.: Harvard University. 1993.

Fitoterapia Warao: una teoría pnéumica de la salud, la enfermedad y la terapia. Caracas: Instituto Caribe de Antropología y Sociología. Fundación La Salle de Ciencias Naturales, 1996.

WILBERT, Werner; AYALA LAFÉE, Cecilia. Los Warao. In: TILLET, Aimé; FREIRE, Germán (Edit.) Salud Indígena en Venezuela. Tomo 2. Caracas: Ministerio del Poder Popular para la Salud, 2007 . D i s p o n i b l e e $n$ : https://www.academia.edu/592203/Salud_Ind\%C3\%ADgena_en_Venezuela_vol_2

WITTFOGEL, Karl. Aspectos del desarrollo de las Sociedades Hidráulicas. In: STEWARD, J.H. (Edit) Las civilizaciones 